\title{
Comparison of Implantation Rates in Ultrasound-guided vs Clinical Touch Embryo Transfer
}

\author{
${ }^{1}$ Manvi Jindal, ${ }^{2}$ ML Swarankar, ${ }^{3}$ Swati Garg, ${ }^{4}$ Urvashi Sharma
}

\begin{abstract}
Aims: The present study was conducted in the fertility unit of a medical college to compare the implantation rate of embryo in ultrasound-guided vs clinical touch method of embryo transfer. Today, approximately $80 \%$ of women undergoing in vitro fertilization/intracytoplasmic sperm injection (IVF/ICSI) will reach the embryo transfer stage with good quality embryos. Traditionally, the 'clinical touch' method has been used to guide placement of the embryo transfer. The use of ultrasound to guide embryo transfer would allow accurate and atraumatic positioning of the catheter tip near the uterine fundus.
\end{abstract}

Materials and methods: A prospective study was conducted at fertility center of Mahatma Gandhi Hospital. A total 100 patients requiring IVF, from a period of July 2011-July 2013 were taken into the study. A total of 50 patients were subjected to USG-guided embryo transfer and 50 patients were subjected to clinical touch embryo transfer. Detailed patient history, clinical examination, relevant investigation and details of procedure were entered in a prestructured proforma. The data were entered and analyzed in Microsoft Office Excel.

Results: There was a significant improvement in the implantation rates in the USG-guided group $(31.9 \%)$ as compared to clinical touch group (24.3\%). There was also a significant improvement in the pregnancy rates in the USG-guided group $(40 \%)$ as compared to the clinical touch group (28\%). Compared with the traditional clinical touch method, the abdominal ultrasound-guided embryo transfer was found to have a number of advantages.

Keywords: Clinical touch embryo transfer, In vitro fertilization, Ultrasound-guided embryo transfer.

How to cite this article: Jindal M, Swarankar ML, Garg S, Sharma U. Comparison of Implantation Rates in Ultrasoundguided vs Clinical Touch Embryo Transfer. J Mahatma Gandhi Univ Med Sci Tech 2016;1(1):10-14

Source of support: None

Conflict of interest: Nil

\section{INTRODUCTION}

Since the first pregnancy using in vitro fertilization (IVF) was achieved nearly 30 years ago many aspects of the

\footnotetext{
${ }^{1}$ Postgraduate, ${ }^{2}$ Professor and Chairperson, ${ }^{3}$ Professor and Head ${ }^{4}$ Assistant Professor

${ }^{1-4}$ Department of Obstetrics and Gynecology, Mahatma Gandhi Medical College and Hospital, Jaipur, Rajasthan, India
}

Corresponding Author: Swati Garg, B-137, Sector-10 Chitrakoot, Ajmer Road, Jaipur, Rajasthan, India, Phone: +919414048000, e-mail: drswati_garg@hotmail.com procedure, such as ovarian stimulation, oocyte recovery, in vitro techniques of fertilization and embryo culture have undergone major revision. ${ }^{1}$ In vitro fertilization technique, in particular, has continued to evolve, the most significant developments being in ovulation induction, the use of intracytoplasmic sperm injection (ICSI) and culture media. $^{2-4}$

In contrast, the technique of embryo transfer (ET) has remained largely unchanged. Today, approximately $80 \%$ of women undergoing IVF/ICSI will reach the embryo transfer stage with good quality embryos. However, only a small proportion will then go on to achieve a clinical pregnancy and fewer will achieve a live birth. This failure may be ascribed to deficiencies in either intrinsic embryo quality or endometrial receptivity, or it could also be due to variation in the technique of embryo transfer. This makes 'Embryo Transfer' the final and the crucial step in success of assisted reproductive techniques (ARTs).

Traditionally, the 'clinical touch' method has been used to guide placement of the transfer catheter to within $\sim 10 \mathrm{~mm}$ from the uterine fundus prior to injection of the embryos. This method is essentially 'blind' and relies on the clinician's tactile senses to judge when the transfer catheter is in the correct position. Some clinicians transfer the embryos at a fixed distance from the external os $(\sim 6 \mathrm{~cm})$; however, this may not take into account variation in cervical length or uterine size.

The use of ultrasound to guide embryo transfer was first discussed by Strickler et al who postulated that this would allow accurate and atraumatic positioning of the catheter tip near the uterine fundus, along with visualization of the transferred fluid containing the embryos. By allowing identification of the cervical canal and endometrial cavity, ultrasound can facilitate atraumatic penetration of the catheter into the uterus, thereby minimizing endometrial trauma. Moreover, its use can confirm that the catheter tip is beyond the internal os of the cervix and placement of the embryos is at the desired level in the endometrial cavity. This can be especially helpful in women where the uterine anatomy may be distorted by fibroids or septae. ${ }^{5}$ For these reasons, ultrasound-guided (USG) embryo transfers have been rated as 'easier' and 'cleaner' by clinicians. 
In the USG-embryo transfer both the insertion and the positioning of the catheter are facilitated by transabdominal ultrasound. The embryos are slowly released when the ultrasound scan shows the catheter to be within $1.5 \mathrm{~cm}$ of the fundus of the uterine cavity. Ultrasound also allows visualization of 'transfer bubble' after the embryo had been expelled.

In the clinical touch group, the embryo are released according the clinicians feeling as to the position of the catheter (i.e., as close to the fundus as possible without touching it).

Other important benefits of USG-embryo transfer includes providing an opportunity to observe the transfer catheter, the air bubble, the endometrial cavity and the endometrial feature. The transferred air bubbles are often considered a marker for the embryo's position in the uterus. By performing the transfer under ultrasonographic guidance, the catheter and air bubble can be precisely located. Based on some studies, catheter insertion at 1.5 or $2 \mathrm{~cm}$ from the fundus is better than insertion at $1 \mathrm{~cm}$ from the fundus. The air bubble position at embryo transfer is relevant to the pregnancy rate.

The present study was conducted in the fertility unit of a medical college to compare the implantation rate of embryo in USG vs clinical touch method of embyro transfer.

\section{MATERIALS AND METHODS}

A prospective study was conducted at fertility center of Mahatma Gandhi Hospital, Jaipur.

A total number of 100 infertile patients presented to our fertility center during the period from July 2011 to July 2013 were included in the study. A total of 50 patients were subjected to USG-embryo transfer and 50 patients were subjected to clinical touch embryo transfer after obtaining informed consent from the patients. Institution Ethical Committee approval was taken before commencing the study.

Informed consent was taken from each patients.

\section{Inclusion Criteria}

- Female patients

- History of primary and secondary infertility requiring IVF-ET.

\section{Exclusion Criteria}

Patients with following condition were excluded:

- Fibroid uterus

- Amenorrhea

- Cervical stenosis.
Detailed patient history, clinical examination, relevant investigation and details of procedure were entered in a prestructured proforma. The data was entered and analyzed in Microsoft Office Excel (Table 1).

\section{OBSERVATIONS AND RESULTS}

Table 1: Patients' details

\begin{tabular}{lll}
\hline & $\begin{array}{l}\text { Clinical Touch } \\
(n=50)\end{array}$ & $\begin{array}{l}\text { Ultrasound- } \\
\text { guided } \\
(n=50)\end{array}$ \\
\hline Mean age in years (SD) & $32.0(4.8)$ & $32.3(5.6)$ \\
Primary infertility (\%) & $62 \%$ & $50 \%$ \\
Mean infertility duration (SD) & $8.4(4.8)$ & $8.4(12.9)$ \\
Cause of infertility & & \\
- Female & 24 & 26 \\
- Male & 16 & 11 \\
- Combined & 5 & 9 \\
- Unexplained & 5 & 4 \\
History of tuberculosis & 24 & 28 \\
History of smoking & 18 & 13 \\
History of hypothyroidism & 20 & 17 \\
\hline
\end{tabular}

Table 2: Clinical touch vs ultrasound - observations and outcomes

\begin{tabular}{lll}
\hline & $\begin{array}{l}\text { Clinical touch } \\
(n=50)\end{array}$ & $\begin{array}{l}\text { Ultrasound- } \\
\text { guided }(n=50)\end{array}$ \\
\hline Chemical pregnancy rate & $21 / 50$ & $28 / 50$ \\
Clinical pregnancy rate & $14 / 50$ & $20 / 50$ \\
Implantation rate & $29 / 90$ & $39 / 83$ \\
Positive b-hCG & & \\
Day 3 embryo transfer & 15 & 19 \\
Day 5 embryo transfer & 6 & 9 \\
No. of G-Sacs implanted & & \\
One & 15 & 16 \\
Two & 7 & 8 \\
Three & 0 & 4 \\
\hline
\end{tabular}

In this prospective study of 100 patients for embryo transfer, 50 patients were allocated to 'clinical touch' group, while the remaining 50 were allocated to 'USGguided' group (Table 2).

Majority of the patients in both the groups belonged to 25 to 29 years age group comprising more than onethird of total patients followed by 30 to 34 years age group which constituted just over a quarter and 35 to 40 years age group which comprised another quarter of the patients (Graph 1). The mean age of patient in the clinical touch group was 32 years with a standard deviation of 4.8 years while the mean age of the patient in the USG-guided group was 32.3 years with a standard deviation of 5.6 years. In the comparison of two groups, the $\mathrm{p}$-value was 0.77 which is not significant. 


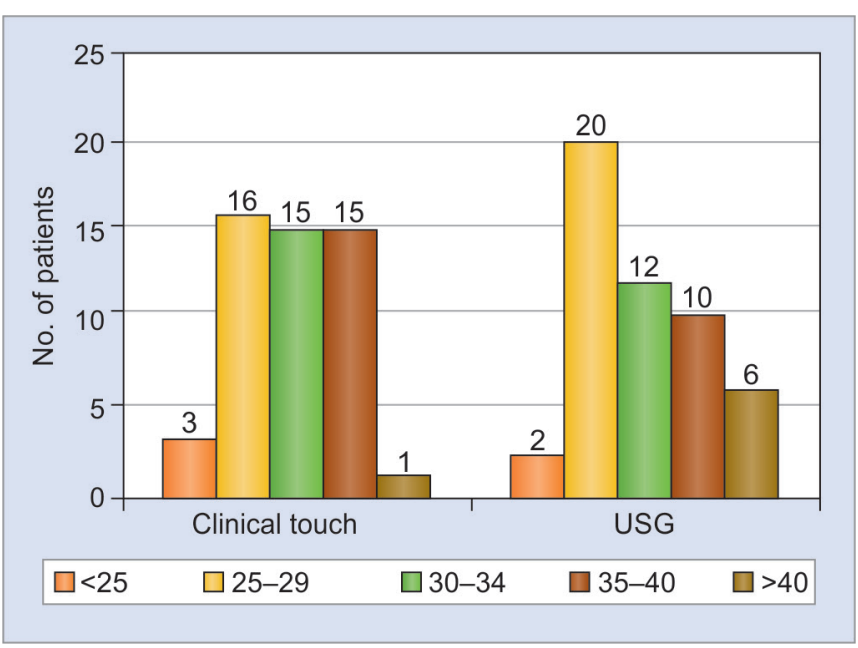

Graph 1: Age distribution of patients in the two groups

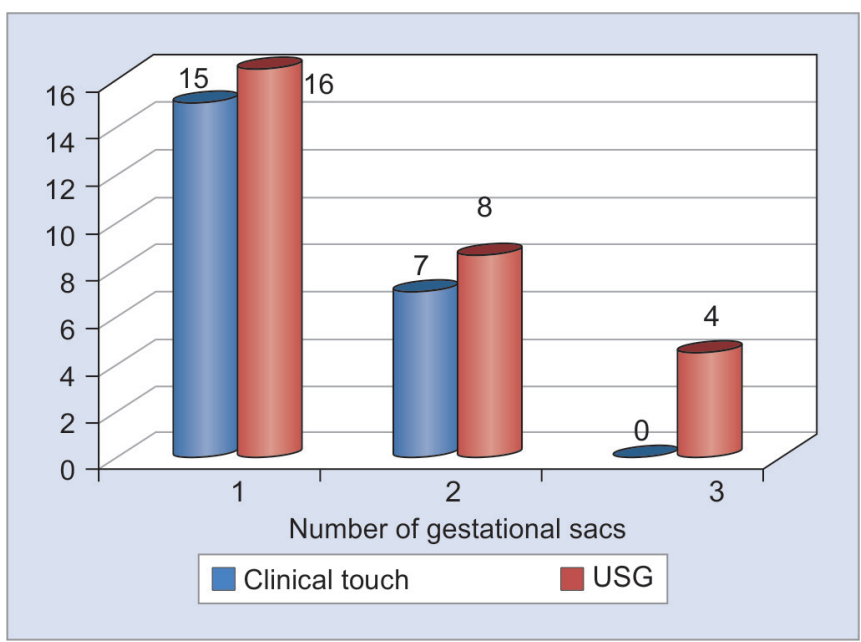

Graph 3: Number of gestational sacs implanted in two groups

Distribution of the patients by duration of infertility revealed an almost equal prevalence between patients presenting with less than 5,6 to 10 years and 11 to 15 years of infertility at almost 30\% each (Graph 2). Overall, the patients in each group presented with a mean of 8.4 years of infertility with a p-value of 1.00 which is not significant.

In the clinical touch group, 15 patients had a single gestational sac implantation and 7 patients had implantation of two gestational sacs (Graph 3). In the USG-guided group, 16 patients had one, eight patients had two and four patients had three gestational sac implantation.

The total numbers of embryos transferred in the clinical touch group were 90, out of which 29 embryos implanted (positive G sac on ultrasound). While in the USG-guided group out of 83 embryos transferred, there were implantation of 39 embryos. Thus the implantation rates in the clinical touch group was $24.3 \%$, while in the USG-guided group was 31.9\% with a p-value of 0.04 which is significant (Graph 4). The results imply the

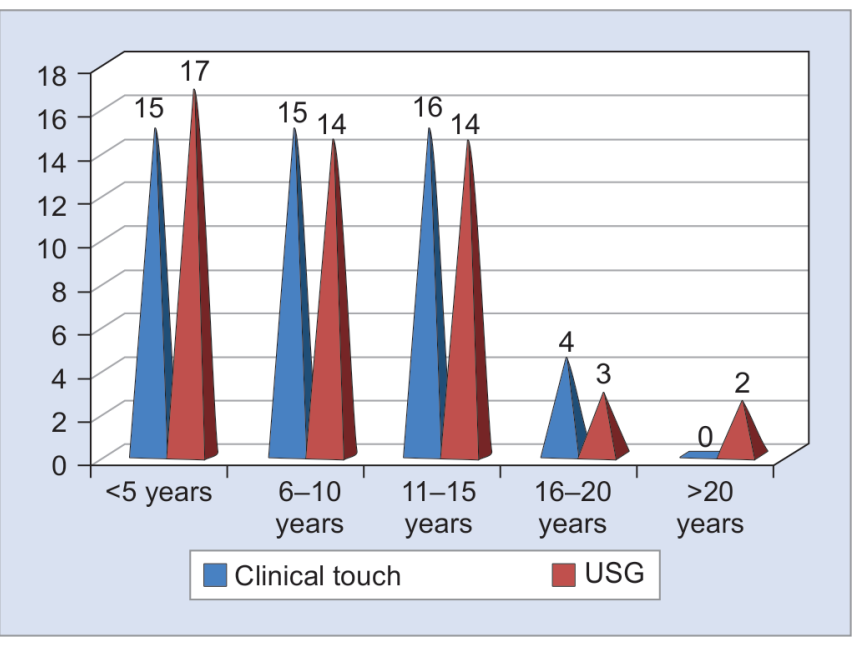

Graph 2: Distribution of patients by duration of infertility in the two groups

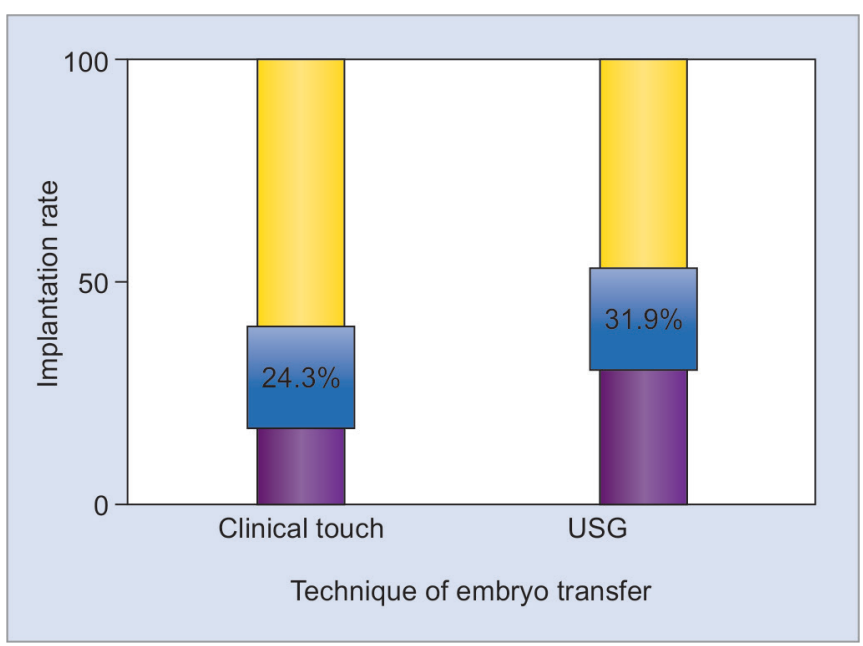

Graph 4: Implantation rates in the two groups

usefulness of ultrasound in the embryo transfer over the clinical touch method.

\section{DISCUSSION}

With the rapid development of ART, ovulation stimulation protocols, ICSI techniques and the embryo culture mediums have been modified greatly. Methods to improve the implantation rate have become a key area of research in the field of ART. The embryo implantation rate in the process of IVF-ET depends on uterine receptivity, quality of embryos and techniques of embryo transfer. Uterine receptivity and the quality of the embryos can be improved by optimization of both the ovarian stimulation protocol and the in vitro culture.

Since the establishment of IVF, embryo transfer has been performed mainly depending on doctors' personal experience and judgment. Experience-based methods have many negative factors that may result in a total faliure of the whole IVF procedure. 
Bolanča et al (2013), reported results of their retrospective study comparing tactile and ultrasound-guided embryo transfer. They proposed that proportion of 'difficult' embryo transfers between tactile and ultrasound group did not show statistically significant differences $\left(12.2 \%\right.$ in tactile, $13 \%$ in ultrasound group, $\chi^{2}=0,053, \mathrm{df}$ $=1, \mathrm{p}>0.01$ ). Proportion of biochemical pregnancies also did not show statistically significant differences $(31.44 \%$ in tactile, $26.73 \%$ in ultrasound group, $\chi^{2}=0,792, \mathrm{df}=1$, $\mathrm{p}>0.01)^{6}$

Brown et al reviewed and evaluated the effectiveness of ultrasound-guided embryo transfer compared with clinical touch the traditional method of embryo transfer. Relevant conference proceedings were also hand searched (ASRM, ESHRE, and FIGO). There was no evidence of a significant difference in the outcome of live birth [OR 1.14 (95\% CI 0.93 to 1.39; $\mathrm{p}=0.02$ ] although heterogeneity was high (64\%). The ongoing pregnancies per woman randomized associated with the ultrasoundguided group (441/1254) was significantly higher than for clinical touch (350/1218) (OR 1.38, 95\%CI 1.16 to 1.64 , $p<0.0003)$. No statistically significant differences in the incidence of adverse events were identified between the comparison groups. ${ }^{7}$

Ali et al studied the implantation, clinical pregnancy and miscarriage rates after introduction of ultrasoundguided embryo transfer and compared the reproductive outcome of USG embryo transfers vs clinical touch embryo transfers. They postulated that implantation rate was significantly higher in the USG embryo transfer group compared with the non-USG embryo transfer group (fresh: 19.9 vs 9.5\%, p < 0.0001; frozen: 13.1 vs $7.3 \%, \mathrm{p}<0.0004)$. The clinical pregnancy rate was also significantly higher in the former group (fresh: 26.9 vs $12.5 \%, \mathrm{p}<0.0001$; frozen: 15.6 vs $8.9 \%$, $\mathrm{p}<0.0015)^{8}$

Eskandar et al reported in their prospective, single operator, randomized, controlled trial comparing ultrasound to clinical touch methods of embryo catheter guidance that live-birth/ongoing pregnancy rate was significantly higher in the ultrasound-embryo transfer group [68/183 (40.98\%)] than the clinical touch embryo transfer group [50/190 (28.42\%)] $(\mathrm{OR}=1.66,95 \% \mathrm{CI}=$ 1.07-2.57). In addition, there was a significantly higher number of clinical pregnancies in the USG-embryo transfer group [75/183 (40.98\%)] than the clinical touch ET group [54/190 (28.42\%)] (OR $=1.75,95 \% \mathrm{CI}=1.14-2.69){ }^{9}$

Drakeley AJ et al results showed that there was no difference in clinical pregnancy or live birth rates between the two groups. The clinical pregnancy rate for ultrasound-guided embryo transfer was $22 \%$ and for nonultrasound-guided embryo transfer was $23 \%$ (odds ratio:
0.96; 95\% confidence interval: 0.79-1.18). They concluded that ultrasound-guided embryo transfer did not improve clinical pregnancy rates and live birth rates in assisted conception. $^{10}$

Kosmas et al reported overall pregnancy rates of $53.3 \%$ (80 patients out of 150 ) and $51.3 \%$ (77 patients out of 150) in the ultrasound-guided and blind ET group respectively, and implantation rates of 32.7 and $34.9 \%$, respectively, whereas both the clinical pregnancy rates were $42 \%$. They concluded that ultrasound-guided ET does not offer any benefit in the hands of an experienced clinician, because similar overall and clinical pregnancy rates were achieved in the blind group. ${ }^{11}$

Li et al reported in their prospective randomized trial on 330 patients that rates of implantation and clinical pregnancy for the USG embryo transfer (19.6-37.1\%, respectively) was significantly higher than for the Clinical Touch controls ( 12.6 and $25 \%$, respectively; $\mathrm{p}<0.05){ }^{12}$

From our data, it is evident that USG embryo transfer significantly improved the pregnancy rates (40 vs 28\%) and implantation rates ( 31.9 vs $24.3 \%$ ). We believe that this apparent improvement can be ascribed to the accurate positioning of the embryo transfer catheter tip near the fundus of the uterus which can be confidently achieved with the use of ultrasound scan guidance. It negates factors, such as inadvertent abutting of the catheter tip against the fundal endometrium or tubal ostia. It has occasionally been observed that the catheter can curl and that the tip would actually be directed toward the cervix without any awareness of this malposition by the clinician.

The procedure was readily accepted by the patients who were reassured by the visualization of the transfer process. The acceptance by the clinicians was also high with no significant added time, and the procedure was done with more confidence as the catheter is advanced to the fundus of the uterus under ultrasound scan guidance.

\section{SUMMARY AND CONCLUSION}

There was a significant improvement in the implantation rates in the USG-guided group (31.9\%) as compared to clinical touch group (24.3\%). There was also a significant improvement in the pregnancy rates in the USG-guided group $(40 \%)$ as compared to the clinical touch group $(28 \%)$.

Compared with the traditional clinical touch method, the abdominal ultrasound-guided embryo transfer has a number of advantages which are follows:

- The doctor can clearly see the position of the uterus, the angle between the body of the cervix and the uterus (version and flexion), the length and direction 
of the uterine cavity and the cervical canal. This helps the gynecologist to choose a suitable catheter for the embryo transfer. With the guidance of ultrasound, the catheter can be bent to follow the axis and can easily pass through the cervical canal, which also avoids over-stimulation and injury of the cervix and uterus.

- The whole process of catheterization and releasing the embryos can be visualized on the ultrasound image, which makes it easier to place the embryos at the correct position.

- Ultrasound-guided embryo transfer significantly decreases the opportunity for stimulation of the uterine cervix and fundus, thereby reducing the chance of the uterine contraction. Excessive uterine contractions at the time of embryo transfer have been associated with a lower clinical pregnancy rate. Stimulation of the cervix causes the release of oxytocin, thus increasing uterine contractility. When the transfer catheter touches the uterine fundus, it induces strong contractions of cervix and uterine fundus. Additionally, avoiding unnecessary operation and removing cervical mucus with soft catheters are of vital importance for the success of embryo transfer.

\section{REFERENCES}

1. Steptoe PC, Edwards RG. Birth after the reimplantation of a human embryo. Lancet 978 Aug 12;2(8085):366.

2. Diedrich K, Felberbaum R. New approaches to ovarian stimulation. Hum Reprod 1998 Jun;13 Suppl 3:1-13.

3. Palermo G1, Joris H, Devroey P, Van Steirteghem Lancet 1992 Jul 4;340(8810):17-18.
4. Gardner DK, Lane M. Culture of viable human blastocysts in defined sequential serum-free media. Hum Reprod 1998; 13(suppl 3):148e159.

5. Hurley VA, Osborn JC, Leoni MA, et al. Ultrasound-guided embryo transfer: a controlled trial. Fertil Steril 1999;55:559-562.

6. Orešković, Majda; Rudančić, Tina; Ljiljak, Dejan; Grubić Kezele, Tanja; Jakovac, Hrvoje. Metallothioneins expression and labile zinc content in spermatozoa of fertile males and subfertile patients with oligoasthenoteratozoospermia Periodicum biologorum/Vitale, Branko (ur.).-Zagreb: Hrvatsko prirodoslovno društvo 2013;64-64.

7. Brown J, Buckingham K, Abou-Setta AM, Buckett W. Ultrasound versus 'clinical touch' for catheter guidance during embryo transfer in women. Cochrane database of systematic reviews, 2010;(1),PP. CD006107, ISSN 1469-493X

8. Ali CR, Khashan AS, Honre G, Fitzgerald CT, Nardo LG. Implantation, clinical pregnancy and miscarriage rates after introduction of ultrasound-guided embryo transfer. Reprod Biomed 2008;17(1):88-93.

9. Eskandar M, Abou-Setta AM, Almushait MA, Mohamed ElAmin, Mohmad SEY. Ultrasound-guidance during embryo transfer: a prospective, single operator, randomized, controlled trial. Fertil \& Steril, Med J Obstet Gynecol 2014;2(4): 1047.

10. Drakeley AJ, Jorgensen A, Sklavounos J, Aust T, Gazvani R, Williamson P, Kingsland CR. A randomized controlled clini trial of 2295 ultrasound-guided embryo transfers. Hum Reprod 2008;23(5):1101-1106.

11. Kosmas IP, Janssens R, Munck L De, Turki HA, Vander Elst J, Tournaye H, Devroey P. Ultrasound-guided embryo transfer does not offer any benefit in clinical outcome: a randomized controlled trial. Hum Reprod 2007;22(5):1327-1334.

12. Li R, Lu L, Hao G, Zhong K, Cai Z, Wang W. Abdominal ultrasound-guided embryo transfer improves clinical pregnancy rates after in vitro fertilization: Experiences from 330 clinical investigations. J Assist Reprod Genet. 2005 Jan;22(1):3-8. 